\title{
Association between Pseudomonas aeruginosa O-antigen serotypes, resistance profiles and high-risk clones: results from a Spanish nationwide survey
}

\author{
Ester del Barrio-Tofiño ${ }^{1}$, Irina Sánchez-Diener ${ }^{1}$, Laura Zamorano ${ }^{1}$, Sara Cortes-Lara ${ }^{1}$, Carla López-Causapé ${ }^{1}$, \\ Gabriel Cabot ${ }^{1}$, Germán Bou ${ }^{2}$, Luis Martínez-Martínez ${ }^{3}$ and Antonio Oliver ${ }^{1 *}$ on behalf of the GEMARA-SEIMCI \\ REIPI Pseudomonas study groupt
}

\begin{abstract}
${ }^{1}$ Servicio de Microbiología, Hospital Son Espases, Instituto de Investigación Sanitaria Illes Balears (IdISBa), Palma de Mallorca, España; ${ }^{2}$ Servicio de Microbiología, Hospital Universitario La Coruña, Instituto Investigación Biomédica A Coruña (INIBIC), A Coruña, España;

${ }^{3}$ Unidad de Gestión Clínica de Microbiología, Hospital Reina Sofía, Departamento de Microbiología, Universidad de Córdoba, Instituto Maimónides de Investigación Biomédica de Córdoba (IMIBIC), Córdoba, España
\end{abstract}

${ }^{*}$ Corresponding author. E-mail: Antonio.oliver@ssib.es

†Members of the GEMARA-SEIMC/REIPI Pseudomonas study group are listed in the Acknowledgements section.

Received 23 April 2019; returned 3 July 2019; revised 8 July 2019; accepted 10 July 2019

\begin{abstract}
Objectives: To evaluate the correlation of O-antigen serotypes with resistance profiles and high-risk clones in a Spanish nationwide survey.

Methods: Up to 30 consecutive healthcare-associated Pseudomonas aeruginosa isolates were collected during October 2017 from each of 51 hospitals (covering all Spanish regions) with a total of 1445 isolates studied. MICs of 13 antipseudomonal agents and MDR/XDR profiles had been previously determined, as well as whole-genome sequences of 185 representative XDR isolates. O-antigen serotypes (01-016) were determined by agglutination using serotype-specific antisera (BioRad). The Pseudomonas aeruginosa serotyper (PAst) program was used for in silico serotyping.
\end{abstract}

Results: The most frequent serotypes were 06 (17.8\%), 01 (15.4\%) and 011 (13.3\%). In contrast, the most frequent serotype among XDR isolates (17.3\%) was 04 (34.1\%), distantly followed by 011 (15.9\%). Within serotypes, XDR phenotypes were more frequent for $\mathrm{O} 12(60.0 \%)$ and $04(57.3 \%)$. The most frequent clone among the XDR isolates was ST175 (40.9\%), followed by CC235 (10.7\%), ST308 (5.2\%) and CC111 (3.6\%). Up to 81.6\% of XDR ST175 isolates typed 04, whereas $18.4 \%$ were non-typeable. 04 genotype was detected in all sequenced $(n=55)$ ST175 isolates. On the other hand, CC235 and ST308 were associated with 011, whereas CC111 was linked to serotype 012.

Conclusions: 04 serotype is linked to the MDR/XDR profile of widespread ST175 (typically only susceptible to colistin, amikacin and the novel combinations ceftolozane/tazobactam and ceftazidime/avibactam) and therefore, after local validation, its detection in the microbiology laboratory might be useful for guiding semi-empirical antipseudomonal therapies and infection control measures in Spanish hospitals.

\section{Introduction}

The growing prevalence of nosocomial infections produced by MDR and particularly XDR Pseudomonas aeruginosa strains is associated with significantly increased morbidity and mortality. ${ }^{1}$ This increasing threat results from the extraordinary capacity of $P$. aeruginosa for developing resistance to nearly all available antibiotics by the selection of mutations in chromosomal genes and from the growing prevalence of transferable resistance determinants, particularly those encoding carbapenemases or ESBLs. ${ }^{2}$ The dissemination of MDR/XDR global strains, the high-risk clones, in multiple hospitals worldwide adds further concern. ${ }^{3}$ Since high-risk clones are associated with defined MDR/XDR profiles, diagnostic approaches to their early detection would be useful for guiding antipseudomonal therapies. The most widely accepted goldstandard technique for the definition of such epidemic clones is MLST. ${ }^{3,4}$ Moreover, WGS provides further relevant information for understanding the dissemination and the resistome of MDR/XDR P. aeruginosa high-risk clones. ${ }^{5-7}$ However, MLST and WGS, and other classical molecular typing techniques such as PFGE, are still time consuming and thus not practical for implementation in the 
current diagnostic microbiology routine for guiding antipseudomonal therapies. On the other hand, O-antigen serotyping is a rapid, simple and cheap procedure that, despite not being as discriminatory as those mentioned above, might be useful for the presumptive detection of at least some MDR/XDR high-risk clones. ${ }^{3,8,9}$ Moreover, a program [Pseudomonas aeruginosa serotyper (PAst)] for in silico serotyping of $P$. aeruginosa isolates from WGS data has recently been developed. ${ }^{10}$ However, large-scale surveys of $\mathrm{O}$ antigen serotypes are very scarce and/or old, and none has been performed so far in Spain. Thus, the objective of this work was to determine the association between $P$. aeruginosa O-antigen serotypes, resistance profiles and high-risk clones, taking advantage of a recent large-scale Spanish nationwide survey of $P$. aeruginosa infections. $^{11}$

\section{Material and methods}

\section{$P$. aeruginosa strain collection}

The collection studied included up to 30 consecutive healthcare-associated non-duplicated (one per patient) $P$. aeruginosa clinical isolates collected during October 2017 from each of the 51 participating hospitals, covering all 17 Spanish regions. ${ }^{11} \mathrm{~A}$ total of 1445 isolates were studied. The distribution of sample types was as follows: respiratory (32.8\%), urine $(23.7 \%)$, soft tissue and osteoarticular (23.1\%), blood culture (5.7\%) and others (14.9\%). MICs of ticarcillin, piperacillin/tazobactam, ceftazidime, cefepime, ceftolozane/tazobactam ( $4 \mathrm{mg} / \mathrm{L})$, ceftazidime/avibactam $(4 \mathrm{mg} / \mathrm{L})$, aztreonam, imipenem, meropenem, ciprofloxacin, tobramycin, amikacin and colistin had been determined by broth microdilution according to EUCAST guidelines (www.eucast.org). EUCAST v 8.1 clinical breakpoints were used for interpretation. Up to 252 (17.4\%) of the isolates met the XDR criteria. ${ }^{12}$ In the previous study, ${ }^{11}$ clonal relatedness among XDR isolates had been initially evaluated by PFGE and one representative XDR isolate from each unique macrorestriction pattern and hospital $(n=185)$ was further analysed through WGS, including MLST and resistome analysis.

\section{O-antigen serotyping}

O-antigen serotypes (01-016) were determined in the 1445 isolates by agglutination using commercially available serotype-specific antisera (BioRad ref. 58901-58916, from 01 to 016). The PAst program ${ }^{10}$ (https://cge.cbs. dtu.dk/services/PAst-1.0/) was used for in silico serotyping of the 185 XDR isolates sequenced. The $\chi^{2}$ test was used for the analysis of the prevalence of serotypes in different subgroups. A $P$ value $<0.05$ was considered statistically significant.

\section{Results}

As shown in Table 1, the most frequent serotypes among the 1445 isolates tested were 06 (17.8\%), O1 (15.4\%) and 011 (13.3\%). Up to $14.5 \%$ of the isolates were non-typeable (no agglutination or polyagglutination with the 16 antigens tested). Significant differences in serotype distribution according to the sample type were not detected (not shown). However, the distribution of serotypes was very different among XDR isolates. Interestingly, the most frequent serotype among XDR isolates (17.4\%) was by far 04 , detected in $34.1 \%$ of the isolates (versus $10.4 \%$ of all isolates $P<0.0001)$, distantly followed by 011 (15.9\%) (Table 1). Although globally not frequent, 012 serotype was also significantly associated with the XDR phenotype (4.8\% versus $1.4 \%, P<0.0001)$. Finally, non-typeability was also significantly associated with XDR isolates (24.2\% versus $15.5 \%, P=0.0007$ ).
Table 1. Distribution of O-antigen serotypes among the complete collection of $P$. aeruginosa isolates as well as those showing an XDR phenotype

\begin{tabular}{|c|c|c|}
\hline Serotype & $\begin{array}{l}\text { Total isolates, } n(\%) \\
\quad(n=1445)\end{array}$ & $\begin{array}{l}\text { XDR isolates, } n(\%) \\
(n=252)^{\mathrm{a}}\end{array}$ \\
\hline 01 & $222(15.4)$ & $13(5.2)^{*}$ \\
\hline $\mathrm{O} 2$ & $58(4.0)$ & $4(1.6)^{*}$ \\
\hline $\mathrm{O} 3$ & $100(6.9)$ & $8(3.2)^{*}$ \\
\hline 04 & $150(10.4)$ & $86(34.1)^{\star \star}$ \\
\hline 05 & $75(5.2)$ & $1(0.4)^{*}$ \\
\hline 06 & $257(17.8)$ & $18(7.1)^{*}$ \\
\hline 07 & $39(2.7)$ & $0(0.0)^{*}$ \\
\hline 08 & $17(1.2)$ & $0(0.0)$ \\
\hline 09 & $20(1.4)$ & $1(0.4)$ \\
\hline 010 & $49(3.4)$ & $3(1.2)^{*}$ \\
\hline 011 & $193(13.3)$ & 40 (15.9) \\
\hline 012 & $20(1.4)$ & $12(4.8)^{\star \star}$ \\
\hline 013 & $3(0.2)$ & $0(0.0)$ \\
\hline 014 & $6(0.4)$ & $0(0.0)$ \\
\hline 015 & $7(0.5)$ & $2(0.8)$ \\
\hline 016 & $20(1.4)$ & $3(1.2)$ \\
\hline Non-typeable & $209(14.5)$ & $61(24.2)^{\star *}$ \\
\hline
\end{tabular}

aStatistically significant $\left(\chi^{2}, P<0.05\right)$ lower $\left(^{*}\right)$ or higher $\left(^{* *}\right)$ prevalence of each serotype among XDR isolates compared with non-XDR isolates.

The distribution of non-MDR/MDR/XDR phenotypes among the main serotypes is shown in Figure S1 (available as Supplementary data at JAC Online). As shown, the serotype more strongly associated with XDR phenotypes was 012 (60.0\% of XDR isolates), closely followed by 04 (57.3\%). The next serotypes were 011 and 016 , with only $20.7 \%$ and $15 \%$ of XDR isolates, respectively. Moreover, all other serotypes yielded XDR rates $<10 \%$. On the other hand, the prevalence of XDR phenotype among non-typeable isolates was $29.2 \%$.

In silico O-antigen serotyping was analysed in the 185 sequenced XDR isolates. Table S1 shows the in vitro and in silico Oantigen serotypes for these strains, together with their STs, susceptibility profiles and resistomes. Agreement between in vitro and in silico serotyping was documented in 125 (67.6\%) of the isolates. Lack of coincidence was mostly caused by isolates non-typeable by conventional serotyping (30.8\%) rather than by serotype discrepancies (1.6\%). Table 2 shows the comparative analysis of in vitro and in silico serotyping in the most frequent XDR clones detected in the study (ST175, CC235, CC111 and ST308). As can be observed, ST175 was clearly associated with 04 serotype; 81.6\% of all ST175 isolates analysed $(n=103)$ were 04 , and the remaining $18.4 \%$ were non-typeable, by in vitro serotyping, whereas all 55 sequenced ST175 isolates showed an 04 in silico serotype. Likewise, CC111 isolates were associated with 012 serotype and CC235 and ST308 with 011. However, some exceptions were documented, such as one ST111 showing an 04 in silico serotype (nontypeable by in vitro serotyping) and a few CC235 isolates showing an 01 serotype by in vitro serotyping. Association of serotypes with susceptibility profiles and resistance mechanisms was not evidenced beyond the link with STs, although the percentage of nontypeable strains was particularly high (40\%) among those producing carbapenemases or ESBLs (Table S1). 
Table 2. In vitro and in silico O-antigen serotypes among the most frequent high-risk clones detected in the study

\begin{tabular}{|c|c|c|c|c|c|c|}
\hline \multirow[b]{2}{*}{ ST } & \multicolumn{3}{|c|}{ In vitro O-antigen serotype } & \multicolumn{3}{|c|}{ In silico O-antigen serotype } \\
\hline & total isolates tested, $n$ & serotype & isolates, $n(\%)$ & total isolates tested, $n$ & serotype & isolates, $n(\%)$ \\
\hline \multirow[t]{2}{*}{ ST175 } & 103 & 04 & $84(81.6)$ & 55 & $\mathrm{O} 4$ & $55(100)$ \\
\hline & & non-typeable & $19(18.4)$ & & & \\
\hline \multirow[t]{3}{*}{ CC235 } & 29 & 011 & $19(65.5)$ & 22 & 011 & $22(100)$ \\
\hline & & 01 & $3(10.3)$ & & & \\
\hline & & non-typeable & $7(24.1)$ & & & \\
\hline \multirow[t]{2}{*}{ CC111 } & 10 & 012 & $6(60)$ & 10 & 012 & $9(90)$ \\
\hline & & non-typeable & $4(40)$ & & 04 & $1(10)$ \\
\hline \multirow[t]{2}{*}{ ST308 } & 13 & 011 & $11(84.6)$ & 9 & 011 & $9(100)$ \\
\hline & & non-typeable & $2(15.4)$ & & & \\
\hline
\end{tabular}

\section{Discussion}

To our knowledge, this is the first large-scale (1445 isolates) nationwide (51 hospitals covering all Spanish regions) survey on the distribution of $P$. aeruginosa O-antigen serotypes among healthcare-associated clinical isolates ever performed worldwide. Using commercially available antisera (BioRad) covering $16(01-$ 016) of the 20 O-specific antigens defined by the International Antigenic Typing Scheme (IATS), ${ }^{9} 14.5 \%$ of the isolates were nontypeable and thus represented a relatively minor issue for the application of this typing scheme. This proportion of non-typeable isolates was indeed lower than that documented in another previous large study (35\%), ${ }^{8}$ likely owing to the overrepresentation of cystic fibrosis (CF) isolates, which are mostly non-typeable owing to the loss of the $\mathrm{O}$-antigen as part of the adaptive process for long-term persistence in chronic infections. Although CF isolates were not excluded in our study, they represented $<1 \%$ of the consecutive isolates included.

Serotypes 06, 01 and 011 were the most frequent among $P$. aeruginosa clinical isolates from Spanish hospitals in our study. Although a comparison with other previous surveys is not straightforward owing to the small number of isolates included or strain selection bias (such as MDR or CF), these three serotypes appear to be those most frequent globally. $8,13,14$

Beyond a typing procedure with low discriminatory capacity in outbreak characterization, $\mathrm{O}$-antigen serotypes have been used as a (likely indirect) marker of strain virulence or clinical outcome. ${ }^{13,15,16}$ However, the likely most prominent feature is the association of certain serotypes with MDR/XDR profiles. While 011 and 012 have been extensively linked to MDR/XDR strains worldwide, $^{3}$ our work shows that the most frequent serotype among Spanish XDR isolates is by far 04, linked to the widespread ST175 high-risk clone. Serotype 012 was also strongly linked to XDR profiles in our study, but the overall prevalence of this serotype, linked to ST111, was very low. On the other hand, despite the fact that our results showed that $\mathrm{O} 11$ was frequent among $P$. aeruginosa isolates from Spanish hospitals, it was not so strongly linked to XDR profiles, even if documented in ST235 and ST308 high-risk clones.

Overall, a high degree of concordance between in vitro and in silico serotyping was documented, but, as previously described, ${ }^{10}$ the latter approach enabled nearly $100 \%$ typeability. Moreover, in silico serotyping detected one O4 ST111 isolate (non-typeable in vitro), whereas all other sequenced isolates from this clone were, as expected, 012. Interestingly, a recent study suggested that in fact ST111 was originally 04 and that it became an epidemic MDR clone following acquisition of 012 determinants and a quinolone resistance mutation in gyrA [C248T (T83I)]. ${ }^{17}$ Indeed, the resistome analysis performed indicated that the single 04 ST111 isolate was the only isolate studied from this clone not showing the GyrA T83I mutation (Table S1). Therefore, this isolate, despite showing an XDR profile, appears to have diverged prior to the emergence of the 012 ST111 epidemic strain. In relation to the potential association between O-antigen serotypes and GyrA mutations, it is noteworthy that the widespread 04 ST175 clone invariably shows a combination of two GyrA mutations (T83I and D87N) (Table S1); the role of this association in the epidemic dissemination of this clone needs to be further explored.

In summary, this large-scale nationwide survey on the distribution of $P$. aeruginosa $\mathrm{O}$-antigen serotypes among healthcareassociated clinical isolates revealed that 04 serotype is very strongly linked to the MDR/XDR profile of widespread ST175 (typically only susceptible to colistin, amikacin and the novel combinations ceftolozane/tazobactam and ceftazidime/avibactam) and therefore its detection in the microbiology laboratory might be useful for guiding semi-empirical antipseudomonal therapies and infection control measures in Spanish hospitals. However, its implementation needs to be adapted to the local epidemiological data (circulating high-risk clones and associated resistance profiles). Likewise, the occurrence of false-positive results should be considered ( $>30 \%$ of 04 isolates were non-MDR) and false-negative results $(20 \%$ of ST175 isolates were classified as nontypeable).Thus, it might be useful to combine O-antigen serotyping with other simple procedures, such as MALDI-TOF biomarker peak analysis, ${ }^{18}$ for the rapid detection of high-risk clones in the clinical microbiology laboratory.

\section{Acknowledgements}

\section{Members of the GEMARA-SEIMC/REIPI Pseudomonas study group}

Fátima Galán, Irene Gracia, Manuel Antonio Rodríguez, Lina Martín, Juan Manuel Sánchez, Laura Viñuela, $M^{a}$ Victoria García, José Antonio Lepe, 
Javier Aznar, Inma López-Hernández, Cristina Seral, Francisco Javier Castillo-García, Ana Isabel López-Calleja, Carmen Aspiroz, Pedro de la Iglesia, Susana Ramón, Elena Riera, María Cruz Pérez, Carmen Gallegos, Jorge Calvo, María Dolores Quesada, Francesc Marco, Yannick Hoyos, Juan Pablo Horcajada, Nieves Larrosa, Juan José González, Fe Tubau, Silvia Capilla, Mar Olga Pérez-Moreno, Ma José Centelles, Emma Padilla, Alba Rivera, Beatriz Mirelis, Raquel Elisa Rodríguez-Tarazona, Noelia Arenal-Andrés, María del Pilar Ortega, Gregoria Megías, Inmaculada García, Cristina Colmenarejo, José Carlos González, Nora Mariela Martínez, Bárbara Gomila, Salvador Giner, Nuria Tormo, Eugenio Garduño, José Andrés Agulla, Alejandro Seoane, Julia Pita, Isabel Paz Vidal, David Mauricio Guzmán, Marta García, María Luisa Pérez del Molino, Gema Barbeito, Fernando Artiles, José Manuel Azcona-Gutiérrez, Yolanda Sáenz, José Antonio Oteo, Ana González, Jennifer Villa, Fernando Chaves, Emilia Cercenado, Teresa Alarcón, Nelly Daniela Zurita, Irene Merino, María Isabel Morosini, Rafael Cantón, María Isabel Sánchez, Laura Moreno, Genoveva Yagüe, José Leiva, José Luis Barrios, Andrés Canut, Jesús Oteo.

\section{Funding}

This work was supported by Merck Sharp and Dohme; Plan Nacional de I + D+i 2013-2016 Instituto de Salud Carlos III, Subdirección General de Redes y Centros de Investigación Cooperativa, Ministerio de Economía, Industria y Competitividad, Spanish Network for Research in Infectious Diseases (REIPI RD16/0016); and grants PI15/00088 and PI18/00076 cofinanced by European Development Regional Fund ERDF 'A way to achieve Europe’, Operative program Intelligent Growth 2014-2020.

\section{Transparency declarations}

This work was partially financed by a grant from Merck Sharp and Dohme to A. O. The funders had no role in design, execution, analysis or reporting of the research.

\section{Supplementary data}

Figure S1 and Table S1 are available as Supplementary data at JAC Online.

\section{References}

1 Juan C, Peña C, Oliver A. Host and pathogen biomarkers for severe Pseudomonas aeruginosa infections. J Infect Dis 2017; 215 Suppl 1: 44-51.

2 Potron A, Poirel L, Nordmann P. Emerging broad-spectrum resistance in Pseudomonas aeruginosa and Acinetobacter baumannii: mechanisms and epidemiology. Int J Antimicrob Agents 2015; 45: 568-85.

3 Oliver A, Mulet X, López-Causapé C et al. The increasing threat of Pseudomonas aeruginosa high-risk clones. Drug Resist Updat 2015; 21-22: 41-59.
4 Curran B, Jonas D, Grundmann $\mathrm{H}$ et al. Development of a multilocus sequence typing scheme for the opportunistic pathogen Pseudomonas aeruginosa. J Clin Microbiol 2004; 42: 5644-9.

5 Kos VN, Déraspe M, McLaughlin RE et al. The resistome of Pseudomonas aeruginosa in relationship to phenotypic susceptibility. Antimicrob Agents Chemother 2015; 59: 427-36.

6 Cabot G, López-Causapé C, Ocampo-Sosa AA et al. Deciphering the resistome of the widespread Pseudomonas aeruginosa sequence type 175 international high-risk clone through whole-genome sequencing. Antimicrob Agents Chemother 2016; 60: 7415-23.

7 Del Barrio-Tofiño E, López-Causapé C, Cabot G et al. Genomics and susceptibility profiles of extensively drug-resistant Pseudomonas aeruginosa isolates from Spain. Antimicrob Agents Chemother 2017; 61: pii: e01589-17.

8 Pirnay JP, Bilocq F, Pot B et al. Pseudomonas aeruginosa population structure revisited. PLoS One 2009; 4: e7740.

9 Lam JS, Taylor VL, Islam ST et al. Genetic and functional diversity of Pseudomonas aeruginosa lipopolysaccharide. Front Microbiol 2011; 2: 118.

10 Thrane SW, Taylor VL, Lund $O$ et al. Application of wholegenome sequencing data for O-specific antigen analysis and in silico serotyping of Pseudomonas aeruginosa isolates. J Clin Microbiol 2016; 54: 1782-8.

11 Del Barrio Tofiño E, Zamorano L, Cortés-Lara S et al. Spanish nationwide survey on Pseudomonas aeruginosa antimicrobial resistance mechanisms and epidemiology. J Antimicrob Chemother 2019; 74: 1825-35.

12 Magiorakos AP, Srinivasan A, Carey RB et al. Multidrug-resistant, extensively drug-resistant and pandrug-resistant bacteria: an international expert proposal for interim standard definitions for acquired resistance. Clin Microbiol Infect 2012; 18: 268-81.

13 Lu Q, Eggimann P, Luyt CE et al. Pseudomonas aeruginosa serotypes in nosocomial pneumonia: prevalence and clinical outcomes. Crit Care 2014; 18: R17.

14 Faure K, Shimabukuro D, Ajayi T et al. O-antigen serotypes and type III secretory toxins in clinical isolates of Pseudomonas aeruginosa. J Clin Microbiol 2003; 41: 2158-60.

15 Mittal R, Sharma S, Chhibber $S$ et al. Correlation between serogroup, in vitro biofilm formation and elaboration of virulence factors by uropathogenic Pseudomonas aeruginosa. FEMS Immunol Med Microbiol 2010; 58: 237-43.

16 Di Martino $P$, Gagnière $H$, Berry $H$ et al. Antibiotic resistance and virulence properties of Pseudomonas aeruginosa strains from mechanically ventilated patients with pneumonia in intensive care units: comparison with imipenemresistant extra-respiratory tract isolates from uninfected patients. Microbes Infect 2002; 4: 613-20.

17 Thrane SW, Taylor VL, Freschi L et al. The widespread multidrug-resistant serotype 012 Pseudomonas aeruginosa clone emerged through concomitant horizontal transfer of serotype antigen and antibiotic resistance gene clusters. MBio 2015; 6: e01396-15.

18 Mulet X, García R, Gayá M et al. O-antigen serotyping and MALDI-TOF, potentially useful tools for optimizing semi-empiric antipseudomonal treatments through the early detection of high-risk clones. Eur J Clin Microbiol Infect Dis 2019; 38: 541-4. 